\title{
THE BIOLOGICAL TROPISMS OF THE CEREBRAL CORTEX
}

\author{
D. DeNNY-Brown *
}

Of the many notable contributions of Dr. Luis Barraquer-Ferré to medicine, we have been especially interested in his studies of the semeiology of the frontal lobe. In these he has followed the lead of the distinguished Barraquer-Roviralta, who in 1921 first described the grasp reflex of the foot. We have found the monograph of Barraquer-Ferré (1946), summarizing his many earlier papers, especially valuable. In paying tribute to his distinguished contributions we would like to discuss his recent description of "Forced Circling Gait in Progressive Circles"' (1939, 1951).

Circling gait to the same side of a frontal lobe lesion in animals has been observed by many experimenters since Bianchi (1895), and usually lasts only three or four weeks after the lesion. For a shorter period it is associated with turning of the head and eyes to the side of the lesion. It also has been well described by Barraquer-Ferré (1933). These symptoms are seldom seen in man, and then usually only for a few days following a severe frontal lesion (Moniz and Lima, 1936). More commonly there are only slight deviations in posture (Goldstein, 1923) and a disinclination to look to the side of the lesion (Foerster, 1936). We have observed that patients keep turning in bed to the side of a severe unilateral frontal traumatic lesion, but by the time they are sufficiently recovered to walk the symptom has disappeared. Disappearance of these effects is evidently a form of compensation, for if in the monkey the remaining frontal lobe is removed, the animal for a time circles to the other side, with brief deviation of the head and eyes to that side.

Associated with circling movement is a general restlessness. This develops later, usually in the second or third week after ablation of the frontal lohe in the monkey. This restlessness takes the form of a continual pacing up and down the cage, often with stereotyped habits such as touching some prominent object such as a feeding box over and over again. This restless behavior becomes gradually less prominent, and after two or three months is only present when the animal is disturbed, or is confined

From the Department of Neurology, Harvard Medical School, and the Neurological Unit, Boston City Hospital.

* Putnam Professor of Neurology, Harvard University. 
to a small cage. In human patients such restlessness takes the form of stereotyped habits, such as patting the bed clothes, arid seldom persist for more than one month.

Of the many effects which can be produced by electrical stimulation of the frontal lobe, turning of the head and eyes to the opposite side is well known (Ferrier, 1886). It is most easily produced by stimulation of the granular cortex of the middle frontal convolution (area 8), but can be obtained from a much wider area by facilitation. Kennard and Ectors (1938) reported that following ablation of area 8 in monkeys deviation of the head and eyes and circling to the side of the lesion occurred. Others, including ourselves, reviewed by Denny-Brown (1951) have found that a much larger frontal lesion is necessary. We are of the opinion that the motor effects from stimulation of area 8 are only the result of a relative concentration in that area of the cortifugal pathway of a general frontal lobe function. When adversive movement of the head and eyes is the first movement in an epileptic convulsion the focus is likely to be in area 8 (Foerster, 1936), and the most interesting circling gait observed by Barraquer-Ferré (1939) exhibits the fully deveioped phenomenon in man, of which the negative aspect had been observed in ablation experiments.

The meaning of these symptoms is a most important question for the philosophy of cerebral function. Many have proposed that these adversive movements mean that the frontal lobes of the brain are concerned with orientation in space. Others have postulated a liaison betwcen frontal lobe, vestibular centers and cerebellum, pointing to the large fronto-pontine tract as its anatomical pathway. The disappearance of the symptoms, though the fronto-pontine tract is completely degenerated, is difficult to explain. Delmas-Marsalet (1936), on the basis of experiments in dogs, concluded that the deviation of the head and eyes set up an altered neck reflex which secondarily led to circling movements. The circling movement, however, is present long after the deviation of the head and eyes has disappeared. Furthermore, lesions of the vestibular system result not in circiing gait but in torsion movements of the head and body round its own axis which are quite different from the mouvement de manege.

Deviation of the head and eyes to the side of the lesion is also seen to follow large lesions of the frontal and cingulate cortex in man. The movement is automatic. It will, however, immediately cease if the natural stimulus is abolished. This is shown in figure 1 , which shows a patient suffering from an extensive left fronto-parietal lesion, resulting from carotid occlusion (left middle and anterior cerebral artery syndrome). $\mathrm{He}$ is aphasic and unresponsive, and has a right hemiplegia. For many months 

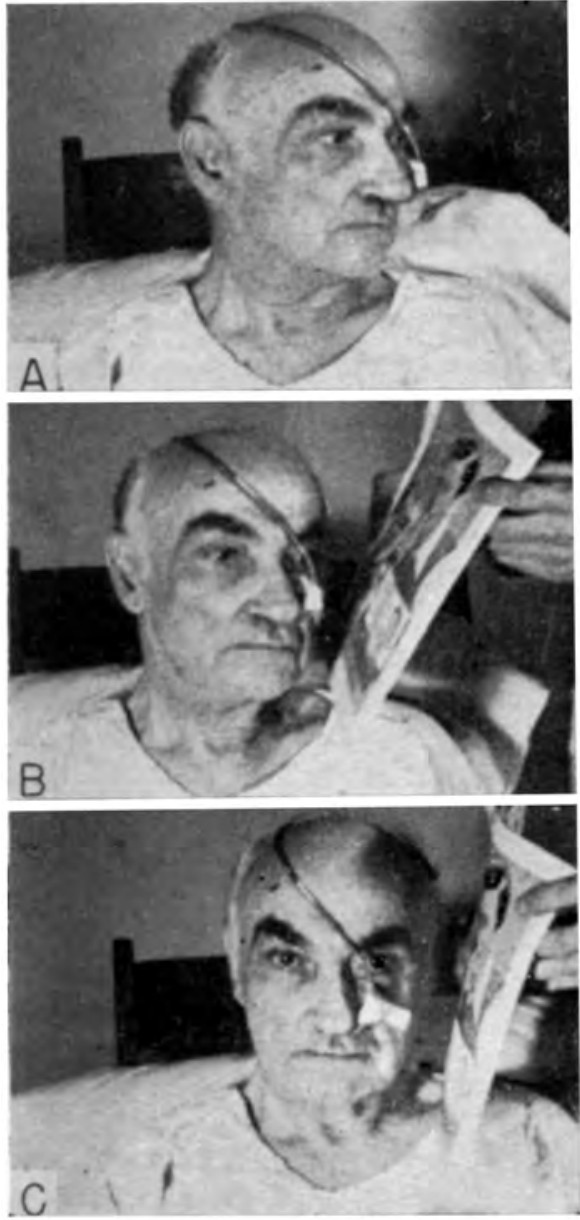

Fig. 1

he lay or sat with the head and eyes turned steadily to the left (fig. 1A). If his vision to the left was blocked, his head and eyes automatically turned slowly to the front (figure $1 B$ and $C$ ). If the obstruction was removed the head and eyes turned slowly to the left again. This response was unfailingly present, and never varied for six months of observation. There was no visual fixation. It is clear that the left visual field as a whole attracted the movement of head and eves. The movement is therefore directly comparable to a biological tropism, and is the result of an attraction of the remaining organism as a whole by the external environment.

In our own work we have been impressed by the instability of these reactions when they result from lesions of the frontal lobe alone. Even the easily produced grasp reflex and forced grasping, which are much more commonly seen in the clinic than forced deviations, undergo progressive modification. Except with very severe lesions, the response is progressively modified, becoming more delicate and more transient. Instinctive grasping gradually becomes transformed into compulsive palpation, and later compulsive touching. The attraction of new objects is still present, but the reaction is brief and repetitive. Seyffarth and Denny-Brown (1948) found that the grasp reflex and instinctive grasping in patients with frontal lobe lesions could be suppressed by the patient, but only by avoiding further stimulation of the palm of the hand. These reactions had not appeared because of inability of the patient to suppress them. How is it, then, that they are absent in the normal? The answer is obvious in the monkey with recent unilateral frontal lobe lesion, for the normal limb has a withdrawal reaction in response to contact, which we have called 
the "avoiding reaction" (Denny-Brown, Saenz-Arroyo and Twitchell, 1950) and which is absent on the side opposite the frontal lobe lesion, where it is replaced by instinctive grasping. The avoiding reaction can be demonstrated in normal man, but his attention is more difficult to distract than that of the monkey. The attentive process of normal consciousness interfers with both reactions in the normal human.

We have further found that the anterior part of the cingulate gyrus is most active in tactile avoiding reactions, which seldom return following its removal, and never return if area 8 is removed in addition. Partial damage restricted to either of these areas results in transient interference with avoiding responses, and in the appearance of instinctive grasping. Instinctive grasping is therefore a "transcortical" release symptom (DennyBrown, 1951). Instinctive grasp reactions are not themselves lost unless the pre- or post-central gyrus are damaged. Following ablation of area 4 these adaptive tactile reactions, including tactile placing, never return. For this reason we regard the rolandic region of the cortex as a stereotactic apparatus for exploring space (Denny-Brown, 1950). Following removal of area 4 the thalamic body-on-body righting reflex is released, which takes the form of a flexion of all the joints of the limb in response to traction on them, modified by contacts with the body surface. It has been called the "grasp reflex" by Fulton (1949) and others. It is important to realize that "grasp-reflex" in the terminology of these authors refers to what in the clinic the neurologist calls "hemiplegic spasticity". In the recovery of hemiplegia in the clinic the return of ability to move the limbs can be shown to depend on the return of first the traction response, and later the grasp reflex, and their successive conversion to willed control by the patient (Denny-Brown, 1951, Twitchell, 1951). The grasp reflex and instinctive grasping as defined by us (Seyffarth and Denny-Brown, 1948) requires the integrity of area 4 . We also differ with Hines (1943) and Fulton (1949) in that we do not find any change in reflex grasping of hand or foot following lesions of area 6. For us the cortical grasping reactions are released by damage to any part of a tactile avoiding mechanism which is widely distributed in the frontal lobe, but more especially in areas 8 and 24 (Denny-Brown, 1951).

We are certain that the cerebral cortex other than area 4 is important in the control of movement. In man the contactual mechanisms of area 4 -are evidently of great importance in movements of tactile exploration and palpation, and the ability to make similar movements freely in space. The spasticity which is associated with loss of these movements from rolandic lesions conceals less important responses to visual and auditory stimulation. We have recently found some evidence of these "extra-rolandic" motor responses in cases of parietal lobe lesion. where involuntary extension of the upper limb with extension of wrist and fingers is commonly 
caused by the presence of an object entering the corresponding visual field (Denny-Brown, Meyer and Horenstein, 1952). The phenomenon in another such case is shown in figure 2 , a patient suffering from an old left hemiplegia, and a recent left parietal lesion. The recent lesion had led to the appearance of involuntary movements in the right hand, as. sociated with loss of position sense and with usiereognosis. Though the resting posture of the right limb was natural (fig. 2A), the hand adopted an extended and pronated attitude when the patient was asked to hold it in front of her (fig. 2B). The pronation was present only when an observer was on the right side of the patient, i.e., in her right field of vision. When the left upper limb was examined by an observer on the left side, the right fingers would exiend voluntarily (fig. 2C). If the observer withdrew, the fingers of the right hand would flex. only io extend again as he reapproached. If the observer requested the patient to use the right hand, for example to grasp the observer's hand, the patient's fingers overextended (fig. 2D). When the patient used the right hand spontaneously, such overextension did not occur. This involuntary extension and pronation is a phenomenon frequently present after lesions of the parietal lobe. We have called the isolated phenomenon (as in fig. 2C) "levitation", for the whole limb may sometimes be raised. This
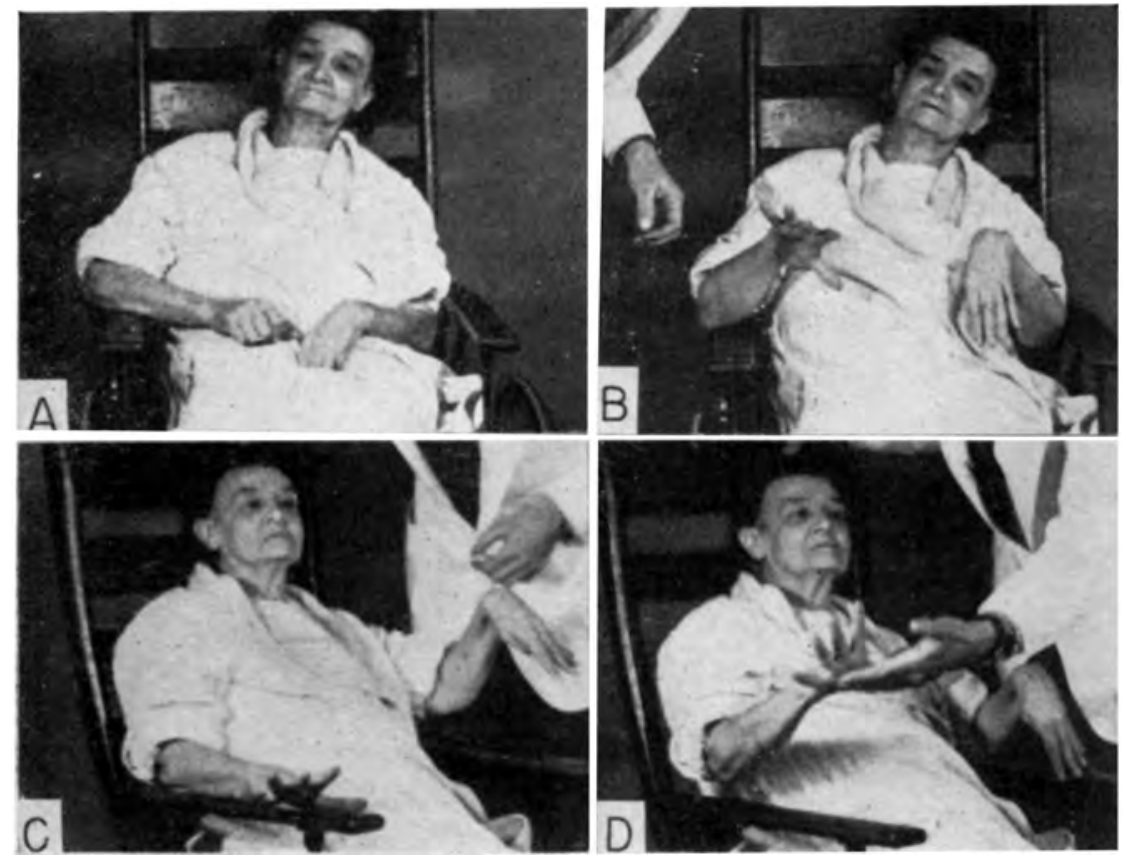

Fig. 2 
is an involuntary release phenomenon, comparable to the instinclive grasp reflex in frontal lobe lesion. Both are due to the transcortical release of cerebral motor automatisms.

We would regard the cerebral control of movement as a mechanism based on tropisms, by which stimuli from the external world attract or repel the whole organism. In the cortex the various exteroceptive sensory systems compete for the dominance of motor behavior. The effect of each sensory system is in equilibrium with the others. Cerebral lesions disturb this relationship in such a way that loss of one effect results in overaction of others (Denny-Brown, 1950, 1951). This disequilibrium. or disintegration, results in the appearance of the instinctive grasp reaction, and of the "levitation" phenomeron. In each instance something is lost, the tactile avoiding reaction in frontal lobe lesion, the visual avoiding reaction in parietal lobe lesion. We are pleased and honored to present an interpretation of the interesting phenomena of which Dr. BarraquerFerré has long realized the importance.

\section{REFERENCES}

Barraquer-Ferré, I. - Contribución al Conocimiento de la Fisiopatología y Semiología de los Lóbulos Frontales, Barcelona, 1946.

Barraquer-Ferré, L. - Acta Psychiat. et Neurol., 26:259, 1951.

Barraquer-Ferré, L. - Rev. Neurol., $1: 485,1933$.

Barraquer-Ferré, I. - Rev. Neurol., 72:160, 1939.

Barraquer-Roviralta, L. - Rev. Neurol., 1:455, 1921.

Bianchi, L. - Brain, 18:497, 1895.

Delmas-Marsalet, P'. - I'Encephale, 31:15, 1936.

Denny-Brown, D. - J. Nerv. Ment. Dis., 112:1, 1950.

Denny-Brown, D. - The Frontai Lobes. Chap. 2 in Modern 'Trends in Neurology, crlited by $A$. Feiling, London, 1951.

Denny-Brown, D.; 'Twitchell, 'I'. a. Saenz-Arroyo, L. - Trans. Amer. Neurol. Ass., z4:1C3, 1949.

Denny-Brown, D.; Meyer, J. S. a. Horenstein, S. - Brain (in press), 1952.

Ferrier, 1). - 'The Functions of the Brain. 2nd. ed., London, 1886.

Foerster, O. - Handbucin der Neurologie. Vol. 6, Berlin, 1936.

Fulton, J. F. - Physiology of the Nervous System. 3rd. ed., I condon and New York, 1949.

Goldstein, K. - Med. Klinik, 19:965, 1006, 1923.

Hines, M. -- Biol. Rrviews, 18:1, 1943.

Kennard, M. A. a. Ectors, I. - J. Neurophysiol., 1:45, 1938.

Moniz, E. a. J_ima, A. - Rev. Neurol., 65:582, 1936.

Seyffarth, H. a. Denny-Brown, D. - Brail, 71:109, 1948.

Twitchell, '1. - Brain, 74:443, 1951. 